\title{
Silencing of miR-134 suppresses seizures in mice
}

A research team in Dublin, Ireland has shown that a specific microRNA (miRNA) is upregulated in the brains of mice and humans with epilepsy, and could represent a new target for interventions to treat this condition. In a study reported in Nature Medicine, David Henshall and colleagues found that silencing of miR-134 after induction of status epilepticus in mice dramatically reduced the subsequent occurrence of both spontaneous seizures and epilepsy-associated pathology.

MiRNAs are noncoding RNA molecules that regulate protein synthesis by binding to mRNAs, and are being implicated in an increasing number of neurological disorders. "MiR-134 was on our shortlist because of work showing that it regulated dendrites," explains Henshall. "This seemed an interesting miRNA to focus on as dendrites are major sites of excitatory synaptic contact and are known to be changed in epilepsy."

Henshall's team generated a mouse model of status epilepticus by injecting the glutamate receptor agonist kainic acid into the amygdala. The resulting mice exhibited increased levels of miR-134 in the CA1 and CA3 regions of the hippocampus ipsilateral to the injection site. Consistent with this observation,
miR-134 was also found to be upregulated in brain tissue from patients who had undergone surgery to treat refractory temporal lobe epilepsy (TLE).

To establish whether the elevated miR-134 levels were pathogenic, the researchers used an miRNA inhibitor known as an antagomir to silence miR-134 in their mouse model. The Ant-134 antagomir was administered via intracerebroventricular injection $1 \mathrm{~h}$ after induction of status epilepticus. "The result was stunning-over $90 \%$ fewer epileptic seizures during 2-week recordings," says Henshall. "Even when we waited 1 or 2 months, the animals were having far fewer seizures."

In parallel with the reduction in seizure frequency, the antagomir was shown to protect against the development of TLEassociated pathology, which includes neuronal loss, gliosis and mossy fibre rearrangment. Intriguingly, the antagomir also produced a reduction in dendritic spine density in CA3 pyramidal neurons -an effect that, the researchers speculate, might contribute to seizure suppression.

"This is the first study to show an effect of a single miRNA on seizures directly," concludes Henshall. "The antagomirs are a very potent antiseizure tool that works in

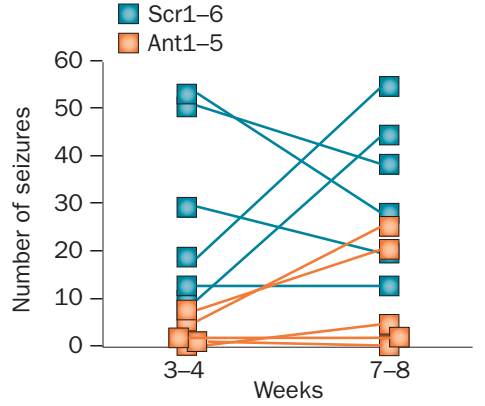

Effects of miR-134 depletion on spontaneous seizure rates in mice 1 month and 2 months post-status epilepticus. Graph shows data from five mice injected with an antagomir targeting miR-134 (Ant1-5) and six mice injected with a nontargeting scrambled sequence (Scr1-6). Image courtesy of D. C. Henshall.

a completely different way from current antiseizure drugs-they could be useful for drug-resistant epilepsy or refractory status." His team now plans to monitor mice beyond 3 months after Ant-134 treatment to establish whether suppression of seizures persists once miR-134 levels have returned to normal.

Heather Wood

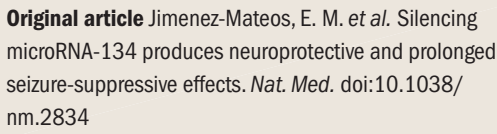
microRNA-134 produces neuroprotective and prolonged seizure-suppressive effects. Nat. Med. doi:10.1038/ $\mathrm{nm} .2834$ 